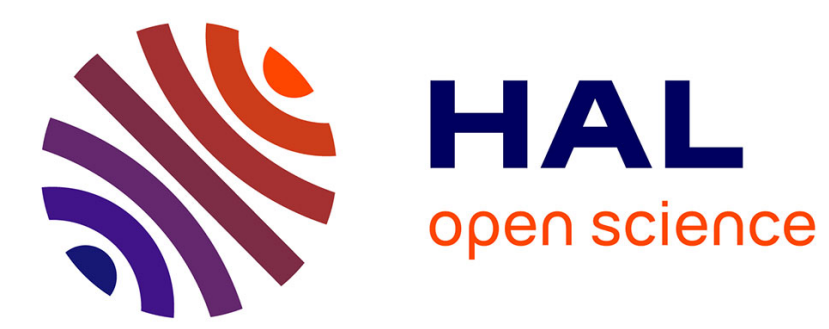

\title{
LA RÉPUBLIQUE DES EXPERTS DANS LA CONSTRUCTION DES LOIS : LE CAS DE LA BIOÉTHIQUE
}

Daniel Borrillo

\section{- To cite this version:}

Daniel Borrillo. LA RÉPUBLIQUE DES EXPERTS DANS LA CONSTRUCTION DES LOIS : LE CAS DE LA BIOÉTHIQUE. Histoire@Politique: revue du Centre d'histoire de Sciences Po, 2011, 2 (14), 10.3917/hp.014.006 . hal-01231982

\section{HAL Id: hal-01231982 \\ https://hal.science/hal-01231982}

Submitted on 21 Nov 2015

HAL is a multi-disciplinary open access archive for the deposit and dissemination of scientific research documents, whether they are published or not. The documents may come from teaching and research institutions in France or abroad, or from public or private research centers.
L'archive ouverte pluridisciplinaire HAL, est destinée au dépôt et à la diffusion de documents scientifiques de niveau recherche, publiés ou non, émanant des établissements d'enseignement et de recherche français ou étrangers, des laboratoires publics ou privés. 


\title{
LA RÉPUBLIQUE DES EXPERTS DANS LA CONSTRUCTION DES LOIS : LE CAS DE LA BIOÉTHIQUE
}

\author{
Daniel Borrillo
}

Presses de Sciences Po | Histoire@Politique

$2011 / 2-n^{\circ} 14$
pages 55 à 83

ISSN 1954-3670

Article disponible en ligne à l'adresse:

http://www.cairn.info/revue-histoire-politique-2011-2-page-55.htm

Pour citer cet article :

Borrillo Daniel, «La République des experts dans la construction des lois : le cas de la bioéthique », Histoire@Politique, 2011/2 n¹4, p. 55-83. DOI : 10.3917/hp.014.006

Distribution électronique Cairn.info pour Presses de Sciences Po.

(C) Presses de Sciences Po. Tous droits réservés pour tous pays.

La reproduction ou représentation de cet article, notamment par photocopie, n'est autorisée que dans les limites des conditions générales d'utilisation du site ou, le cas échéant, des conditions générales de la licence souscrite par votre établissement. Toute autre reproduction ou représentation, en tout ou partie, sous quelque forme et de quelque manière que ce soit, est interdite sauf accord préalable et écrit de l'éditeur, en dehors des cas prévus par la législation en vigueur en France. II est précisé que son stockage dans une base de données est également interdit. 
Daniel Borrillo, «La République des experts dans la construction des lois : le cas de la bioéthique », Histoire@Politique. Politique, culture, société, nº 14, mai-août 2011, www.histoire-politique.fr

\title{
La République des experts dans la construction des lois : le cas de la bioéthique
}

\author{
Daniel Borrillo
}

L'expertise comme légitimation dans la prise des décisions politiques se caractérise par l'appel à l'autorité scientifique en tant qu'instance susceptible de dirimer d'éventuels conflits d'intérêts. Elle agit par un mécanisme de soustraction de la délibération démocratique de certaines questions sensibles, présentées comme impliquant des valeurs communes fondamentales telles que la dignité de la personne humaine, la cohésion sociale ou l'intégrité psychique des individus. Paradoxalement, en matière bioéthique, ce ne sont pas les sciences naturelles qui se sont instaurées en instance expertale lors de la construction des lois, mais les sciences humaines, conçues non pas comme une connaissance sur l'humain, mais plutôt comme un savoir sur l'humanité. Pensé alors comme une limitation des sciences médicales et de la recherche sur le vivant, le dispositif bioéthique fut rapidement investi par l'expertise en sciences humaines. La bioéthique est devenue un savoir normatif, ayant pour but la construction des bornes à l'activité techno-scientifique au détriment du développement d'une dimension plus descriptive et informative. Ainsi, face au danger du scientisme, la bioéthique, en tant que science officielle de protection morale du vivant, a produit un nouveau danger, celui d'une métaphysique des corps et de la vie qui ne fait que transposer, dans une forme laïque, la même idéologie de la tradition catholique.

Dans cet article, nous analyserons le rôle de l'expertise en sciences humaines lors de la dernière révision des lois de bioéthique afin de montrer comment, à partir d'une rhétorique humaniste, une certaine expertise en sciences sociales produit une naturalisation de la famille hétérosexuelle et une justification de l'indisponibilité du corps et de la vie par les individus eux-mêmes concernés.

\section{Le dispositif bioéthique}

Conçue dans les années 1960 comme un champ d'analyse morale, la bioéthique est devenue en peu de temps une discipline prescriptive visant à réguler les effets de l'activité des sciences de la vie et de la santé. Cette dimension normative se prête tout particulièrement à l'intervention de l'expertise comme fondement de légitimité de la prise des décisions législatives. 
Daniel Borrillo, «La République des experts dans la construction des lois : le cas de la bioéthique», Histoire@Politique. Politique, culture, société, nº 14, mai-août 2011, www.histoire-politique.fr

Les dilemmes moraux suscités par certaines pratiques controversées de la science ont rendu nécessaire le développement d'un savoir susceptible d'informer sur l'état d'avancement des recherches scientifiques, de faire le point sur les implications des expériences déjà réalisées, de mesurer les enjeux sociaux et d'établir un corpus de règles permettant d'unifier les pratiques scientifiques impliquant le vivant humain. La bioéthique apparaît ainsi comme une discipline à double dimension, spéculative et prescriptive. Elle est à la fois une réflexion sur les problèmes moraux liés à la recherche sur le vivant et à ses applications, et un guide de conduite des professionnels et des usagers du système de santé. Dans sa dimension normative, le champ bioéthique a été influencé par deux logiques opposées: l'éthique de responsabilité et l'éthique des convictions. Alors que cette dernière privilégie les principes immuables provenant principalement des religions (comme la prohibition de l'avortement et de l'euthanasie au nom de la sacralité de la vie), l'éthique de la responsabilité favorise, au moment de prendre une décision, les conséquences pratiques de l'action. Comme le notait Weber : « Le chrétien fait son devoir et en ce qui concerne le résultat de l'action il s'en remet à Dieu» et «lorsque les conséquences d'un acte fait par pure conviction sont fâcheuses, le partisan de cette éthique n'attribuera pas la responsabilité à l'agent, mais au monde, à la sottise des hommes ou encore à la volonté de Dieu qui a créé les hommes ainsi (...). Au contraire le partisan de l'éthique de responsabilité comptera justement avec les défaillances communes de l'homme (...) et il estimera ne pas pouvoir se décharger sur les autres des conséquences de sa propre action pour autant qu'il aura pu les prévoir ${ }^{1}$. » Ainsi, pour l'éthique de responsabilité, de manière limitée et sous certaines conditions, la liberté de la femme doit primer la vie de l'embryon et celle du malade l'obligation de soins du médecin. Les personnes sont donc tenues pour responsables de leur choix et la loi ne doit intervenir que lorsqu'un comportement lèse un tiers. En fonction de ces deux principes - autonomie individuelle et absence de dommage à autrui - les prohibitions apparaissent toujours comme le résultat d'une délibération démocratique fondée sur des arguments rationnels.

En revanche, pour l'éthique des convictions, ce n'est pas tant le consentement, l'absence de préjudice ou le «consensus du moindre mal » qui constitue le gage de légitimité d'une action mais plutôt l'obéissance à des principes transcendants. Cette soumission apparaît comme la seule garantie de moralité, indépendamment des conséquences pratiques qu'une telle obéissance aveugle serait susceptible de produire.

Le débat bioéthique et ses effets pratiques ne sont très souvent que le reflet de la tension entre ces deux logiques antinomiques et, malgré le caractère laïque des sociétés contemporaines, l'appel plus ou moins explicite à des principes transcendants se substitue souvent à l'argumentation et à la délibération démocratique. Ainsi, la notion de « dignité humaine » apparaît, en France, comme un principe absolu s'imposant à tous, y compris contre la volonté de l'individu qu'il est censé protéger ${ }^{2}$. La confusion entre eugénisme d'État, toujours condamnable, et

\footnotetext{
${ }^{1}$ Max Weber (1919), Le Savant et le politique, Paris, Plon, 1995.

2 Pour une critique de l'utilisation politique de la notion de dignité humaine, voir Olivier Cayla, « Dignité humaine, le plus flou des concepts », Le Monde, 31janvier 2003.
} 
Daniel Borrillo, «La République des experts dans la construction des lois : le cas de la bioéthique», Histoire@Politique. Politique, culture, société, nº 14, mai-août 2011, www.histoire-politique.fr

pratiques de dépistages prénataux (partiellement autorisées) crée un climat culpabilisateur envers des femmes qui décident de mettre fin à une grossesse pouvant aboutir à la naissance d'un enfant handicapé. De même, l'invocation de l'ordre symbolique mis en avant pour s'opposer, entre autres, à la filiation homoparentale ${ }^{3}$, ou encore l'assimilation de l'euthanasie active à un homicide volontaire avec préméditation ne font qu'entretenir un climat de paternalisme d'État enclin à se substituer à la volonté des individus concernés.

Les préoccupations philosophiques relatives aux interventions sur l'humain, qui remontent à la nuit des temps et (elles furent cristallisées dans le Sermon d'Hippocrate au IVe siècle avant J .-C. et plus récemment dans les codes de Persival - 1833 - et de Beaumont - 1847), constituent une règle déontologique, c'est-à-dire une autolimitation de la corporation des médecins. La bioéthique, quant à elle, apparaît comme une intervention extérieure embrassant une palette bien plus large d'acteurs sociaux (chercheurs, ONG, politiciens, avocats...) et va au-delà des actes purement médicaux. En effet, la bioéthique cherche à concilier des intérêts opposés. La tension entre la liberté de la recherche et le respect de certains principes (intégrité physique de la personne, non-souffrance des animaux, non-commercialisation $d u$ patrimoine génétique...) nécessite souvent l'intervention de la loi afin de garantir l'épanouissement de l'ensemble des intérêts légitimes en jeu. En France, la bioéthique s'est posée comme un savoir d'experts malgré l'habillage pluraliste et démocratique que les autorités publiques ont souhaité lui octroyer. La bioéthique surgit comme une préoccupation de l'État vis-à-vis des implications morales du développement des sciences, surtout après la naissance du premier enfant conçu par fécondation artificielle et plus tard par la mise au point des techniques de congélation embryonnaire. Cette dimension publique trouve ses racines dans les «Assises nationales sur la recherche » de 1982, organisées par J ean-Pierre Chevènement (à l'époque ministre de la Recherche) et Philippe Lazar. En 1983, à la suite desdites assises, voit le jour le Comité consultatif national d'éthique pour les sciences de la vie et de la santé $(\mathrm{CCNE})^{4}$. Composé des personnalités issues des différentes corporations (administratives, scientifiques, théologiques, académiques...), le CCNE a pour mission de donner des avis sur les problèmes éthiques et les questions de société soulevés par les progrès de la connaissance dans les domaines de la biologie, de la médecine et de la santé. Le président du CCNE (et ses membres) sont nommés par le président de la République sur désignation de l'administration, après proposition des églises ou des institutions académiques. Le CCNE apparaît ainsi comme une instance d'expertise particulièrement puissante grâce à la légitimité octroyée par l'administration et à la connaissance de l'humain qu'il concentre.

Quelques années plus tard, le Conseil d'État est associé à la réflexion bioéthique, confirmant ainsi l'interventionnisme étatique dans la matière. Dans un rapport du 25 mars 1988, la plus haute juridiction administrative propose un certain nombre de

\footnotetext{
3 Pour une critique de l'utilisation politique de la notion d'ordre symbolique, voir Marcela Iacub, Le Crime était presque sexuel (ch. 16 « Le couple homosexuel, le droit et l'ordre symbolique»), Paris, Flammarion, 2002, p. 347 et suiv.

4 Décret du 23 février 1983.
} 
Daniel Borrillo, «La République des experts dans la construction des lois : le cas de la bioéthique», Histoire@Politique. Politique, culture, société, nº 14, mai-août 2011, www.histoire-politique.fr

solutions juridiques aux problèmes éthiques posés par les nouvelles techniques médicales: procréation assistée, expérimentation humaine, statut de l'embryon, diagnostic prénatal, expérimentation du médicament, banques de données médicales ${ }^{5}$. Le Conseil d'Etat influence depuis la rédaction des lois bioéthiques et ses révisions participant à « l'ère d'acharnement législatif 6 ». Cette ère est dominée par l'omniprésence des rapports officiels au sein desquels l'expert en humanité jouit d'une place éminente.

Cette même année 1988 a été adoptée la loi Huriet-Serusclat, relative à la protection des personnes dans la recherche biomédicale. Elle permet d'encadrer les essais cliniques sur l'humain ${ }^{7}$. La Commission nationale consultative des droits de l'homme $(\mathrm{CNCDH})$, saisie par le gouvernement d'un avant-projet de loi concernant les questions bioéthiques, a rendu, le 21 septembre 1989, un avis dans lequel elle considère, à l'unanimité, qu'une prise de position du législateur est nécessaire afin de soumettre toute recherche et toute intervention sur l'humain aux principes suivants : respect de la dignité de la personne et de son corps, non-patrimonialité du corps et de ses organes ou produits, exigence d'un consentement libre et éclairé de la personne à toute intervention la concernant et refus de toute pratique eugéniste ${ }^{8}$. En 1991, Noëlle Lenoir et Bruno Sturlese rendent un nouveau rapport au Premier ministre ${ }^{9}$ dans lequel ils dressent un panorama international du droit et des pratiques de la biomédecine du début à la fin de l'existence humaine. Les auteurs proposent également des principes directeurs pour une loi-cadre parmi lesquels celui de prendre des mesures législatives sur la non-commercialité du corps humain, la technique des empreintes génétiques et la procréation médicalement assistée. L'année 1992 a été particulièrement prospère avec la publication de deux rapports parlementaires sur la bioéthique, l'un du député Bernard Bioulac ${ }^{10}$ et l'autre du sénateur Franck Sérusclat ${ }^{11}$, ainsi qu'un un nouvel avis de la $\mathrm{CNCDH}^{\text {, où la }}$ Commission réitère l'urgence de légiférer dans la matière. Depuis, les rapports officiels se sont multipliés : l'Inspection générale des Affaires sociales a rendu un avis, en mai 1993, sur le recueil et l'utilisation des tissus d'origine humaine ${ }^{12}$. Au même moment, l'Unesco a mis en place un Comité international de bioéthique composé de trente-six experts indépendants. Ils ont pour mission d'encadrer les progrès des recherches dans les sciences de la vie et leurs applications en veillant au respect des principes de dignité et de liberté de la personne humaine.

\footnotetext{
5 «Sciences de la vie: de l'éthique au droit», Rapport du Conseil d’État, Paris, La Documentation française, 1988.

6 Muriel Flis-Trèves, Dominique Mehl et Evelyne Pisier, « Contre l'acharnement législatif », Pouvoirs, n 56, 1991, « Bioéthique », p. 121.

${ }^{7}$ Loi n 88-1138 du 20 décembre 1988.

8 « Avis sur les sciences de la vie et les droits del'homme », CNCDH 21/ 12/ 1989.

9 « Aux frontières de la vie : une éthique biomédicale à la française », Paris, La Documentation française, 1991.

10 « Rapport d'information sur la bioéthique déposé par la Commission des affaires culturelles, familiales et sociales et la Commission des lois constitutionnelles, de la législation et de l'administration générale de la République, enregistré à la présidence de l'Assemblée nationale le 18 février 1992 », n 2565.

11 « Sciences de la vie et droits de l'Homme : bouleversement sans contrôle ou législation à la française », 1992.

12 Rapport « La vie en questions : pour une éthique biomédicale », 15 novembre 1993.
} 
Daniel Borrillo, «La République des experts dans la construction des lois : le cas de la bioéthique», Histoire@Politique. Politique, culture, société, nº 14, mai-août 2011, www.histoire-politique.fr

Après une ardente discussion d'un an et demi, les trois projets de lois sur la bioéthique ont été adoptés par le Parlement : loi n 94-548 du 1er juillet 1994 relative au traitement des données nominatives ayant pour fin la recherche dans le domaine de la santé et modifiant la loi $n^{\circ} 78-17$ du 6 janvier 1978 relative à l'informatique, aux fichiers et aux libertés ; loi $n^{\circ}$ 94-653 du 29 juillet 1994 relative au respect du corps humain ; loi $n^{\circ}$ 94-654 du 29 juillet 1994 relative au don et à l'utilisation des éléments et produits du corps humain, à l'assistance médicale à la procréation et au diagnostic prénatal. Ces textes, qu'on a communément regroupés sous la dénomination de « lois bioéthiques », constituent la base de la régulation juridique nationale.

\section{Les lois bioéthiques de 1994}

Adopté grâce à une majorité des voix socialistes et avec l'abstention du parti communiste, les lois bioéthiques sont validées par le Conseil constitutionnel le 29 juillet 1994. La France devient ainsi le premier pays au monde à disposer d'un arsenal législatif assez exhaustif se situant entre le libéralisme «anglo-espagnol » et l'interventionnisme germanique. En effet, contrairement au Royaume-Uni et à l'Espagne où la procréation médicalement assistée (PMA) est ouverte aux femmes célibataires (et l'insémination post mortem autorisée), la France interdit cette pratique et limite la PMA aux couples hétérosexuels stériles. Les mères porteuses et les contrats de gestation pour autrui sont également sanctionnés, contrairement à la Grande-Bretagne qui les autorise sous condition de gratuité. Les lois françaises se veulent toutefois plus libérales que la législation allemande (fortement conditionnée par le passé de ce pays) dans laquelle les dons de gamètes, le diagnostic préimplantatoire et toute recherche sur l'embryon constituent des pratiques encadrées sur le plan pénal.

Avec la loi 88-1138 relative à la protection des personnes qui se prêtent à des recherches biomédicales, les lois de 1994 forment un dispositif étendu visant la protection du corps humain, celle des données nominatives en matière de recherche médicale ainsi que la régulation de la circulation des produits d'origine humaine, l'assistance médicale à la procréation et le diagnostic préimplantatoire. Ces lois introduisent dans le Code civil et le Code de la santé publique un certain nombre de principes (corps hors commerce, gratuité des produits humains, consentement éclairé des patients, secret professionnel, droit à l'information, non-brevetabilité du génome) et de règles relatives aux bonnes pratiques scientifiques et médicales, en particulier lorsqu'elles impliquent le vivant (humain et non humain). Les lois bioéthiques mettent en place également un dispositif pénal visant à sanctionner notamment les interventions médicales sans le consentement du malade, les inséminations artificielles en dehors des procédures établies par la loi, l'achat d'embryons humains et les actes de cruauté envers un animal domestique.

La première révision de la loi, fruit de l'expertise internationale et nationale

Dans une recommandation du 13 février 1997, le Conseil de l'Europe établit les principes du traitement des données médicales qui serviront de base pour les futures 
Daniel Borrillo, «La République des experts dans la construction des lois : le cas de la bioéthique», Histoire@Politique. Politique, culture, société, nº 14, mai-août 2011, www.histoire-politique.fr

législations nationales des pays membres ${ }^{13}$. Plus tard, la signature de la Convention pour la protection des droits de l'homme et de la dignité de l'être humain à l'égard des applications de la biologie et de la médecine constitue une étape décisive dans l'internationalisation de la bioéthique. La Convention du Conseil de l'Europe du 4 avril 1997 réaffirme, dans son article 5, le principe selon lequel « une intervention dans le domaine de la santé ne peut être effectuée qu'après que la personne concernée y a donné son consentement libre et éclairé. Cette personne reçoit préalablement une information adéquate quant au but et à la nature de l'intervention ainsi que quant à ses conséquences et ses risques. La personne concernée peut, à tout moment, librement retirer son consentement ».

L'annonce, par une équipe de chercheurs britanniques, le 23 février 1997, de la naissance d'une brebis clonée a constitué l'événement autour duquel s'articule dorénavant le débat bioéthique. L'Organisation mondiale de la Santé (OMS) adopte toute de suite après une résolution condamnant le clonage humain. Elle « affirme que l'utilisation du clonage pour reproduire des êtres humains n'est pas acceptable sur le plan éthique et est contraire à l'intégrité de la personne humaine et à la morale ». La différence entre clonage humain et clonage animal constitue la ligne de partage de l'ensemble des recommandations nationales et internationales, seul le premier est susceptible d'une réprobation morale. Dans un avis du 28 mai 1997, le Groupe européen d'éthique, des sciences et des nouvelles technologies sur les «aspects éthiques des techniques de clonage » considère légitime le clonage des animaux d'élevage, à condition que le but poursuivi et les méthodes employées soient éthiquement justifiés, et moyennant le respect des conditions éthiques, notamment celles consistant à limiter les souffrances de l'animal.

La Déclaration universelle sur le génome humain de l'Unesco condamne formellement dans son article 11 «des pratiques qui sont contraires à la dignité humaine, telles que le clonage à des fins de reproduction d'êtres humains ${ }^{14} \ldots{ }^{\prime}$. En revanche, le clonage reproductif ne constitue pas, pour cet instrument international, une pratique prohibée.

En Europe, après plus de dix ans de débat, la Commission a finalement adopté une directive sur la protection juridique des inventions biotechnologiques ( $n^{\circ}$ 98/44/ CE du 6 juillet 1998) selon laquelle le corps humain, les procédés de clonage de l'être humain et l'intervention génique germinale sur l'homme ne peuvent pas être protégés par la technique du brevet. Dans le même sens, la Charte des droits fondamentaux de l'Union européenne (2000) ratifie, dans son article 3, le principe d'intégrité de la personne dans le cadre de la médecine et de la biologie. Quelques années plus tard, l'Association médicale mondiale adopte une nouvelle résolution sur la noncommercialisation des éléments humains de reproduction ${ }^{15}$ exhortant les gouvernements à promulguer des lois interdisant les transactions commerciales

\footnotetext{
13 « Recommandation R (97) 5 du Comité de Ministres aux États membres relative à la protection des données médicales », Conseil de l’Europe, 1997.

14 « Déclaration universelle sur le génome humain et les droits de l'Homme », 11 novembre 1997.

15 «Résolution de l'AMM sur la non-commercialisation des éléments humains de reproduction » adoptée par la 54 Assemblée générale de l'AMM (Association médicale mondiale), Helsinki, septembre 2003.
} 
Daniel Borrillo, «La République des experts dans la construction des lois : le cas de la bioéthique», Histoire@Politique. Politique, culture, société, nº 14, mai-août 2011, www.histoire-politique.fr

d'ovules, de spermes et d'embryons humains. Depuis le nombre de textes internationaux et européens relatifs au traitement des éléments du corps ne cesse de se multiplier. La première révision des lois françaises a eu lieu dans ce contexte de production des normes internationales, qui ont exercé une influence indéniable sur la réforme nationale.

En France, certaines dispositions des lois bioéthiques sont rapidement considérées comme trop restrictives, et, par conséquent, menaçantes pour le développement de la recherche. Dans deux avis du 11 mars 1997, le CCNE recommande d'assouplir le dispositif légal, en particulier ce qui concerne la recherche sur l'embryon humain, compte tenu des « importantes perspectives dans les recherches thérapeutiques ». De même, la distinction entre clonage reproductif et clonage thérapeutique se consolide avec le rapport du Conseil d'État du 25 novembre 1999 selon lequel seul le clonage reproductif devrait être interdit. Le Conseil d'État demande donc au législateur d'autoriser la recherche sur les cellules souches embryonnaires «qui ouvrent des perspectives thérapeutiques nouvelles en réponse à des maladies ou des handicaps actuellement incurables ${ }^{16}$ ». L'année suivante, un rapport du député Alain Claeys et du sénateur Claude Huriet sur «le clonage, la thérapie cellulaire et l'utilisation thérapeutique des cellules embryonnaires ${ }^{17}$ » renouvelle la condamnation du clonage reproductif et invite à ouvrir le débat sur le clonage thérapeutique «dont de nombreux scientifiques soulignent l'intérêt qu'il pourrait revêtir dans l'exploitation des ressources médicales offertes par les cellules souches pluripotentes qui pourraient être utilisées dans le traitement des diverses maladies dues à des lésions cellulaires (Parkinson, diabète, dystrophie musculaire...) ». L'autorisation du clonage thérapeutique au Royaume-Uni donne lieu à un débat passionné en Europe qui aboutit à une résolution radicale du Parlement européen du 7 septembre 2000 prônant l'interdiction de toute recherche sur le clonage humain et la mise en ouvre de sanctions pénales en cas d'infraction.

Bien que l'avant-projet de loi visant à réviser les lois de 1994, présenté par Lionel Jospin le 28 novembre 2000, prévoyait l'autorisation du clonage thérapeutique, celui-ci est finalement interdit dans la version définitive de la loi adoptée en 2004. En effet, l'article 21 précise : «Est interdite toute intervention ayant pour but de faire naître un enfant génétiquement identique à une autre personne vivante ou décédée. La recherche sur l'embryon humain est interdite». Par dérogation, les recherches peuvent être autorisées sur l'embryon et les cellules embryonnaires. Ne peuvent être concernés que les « embryons conçus in vitro dans le cadre d'une assistance médicale à la procréation qui ne font plus l'objet d'un projet parental » (embryons dits "surnuméraires"). Par dérogation, «le diagnostic biologique [préimplantatoire] effectué à partir de cellules prélevées sur l'embryon in vitro peut également être autorisé, à titre expérimental », pour guérir un enfant atteint d'une maladie

\footnotetext{
${ }^{16}$ Conseil d’État, Les lois de bioéthique, cinq ans après, Paris, La Documentation française, 1999, p. 151
} ${ }_{17} \mathrm{~N}^{\circ} 2198$, du 24 février 2000, Assemblée nationale. 
Daniel Borrillo, «La République des experts dans la construction des lois : le cas de la bioéthique », Histoire@Politique. Politique, culture, société, nº 14, mai-août 2011, www.histoire-politique.fr

génétique incurable grâce à la naissance d'un enfant indemne (bébés dits "du double espoir" ou "bébés-médicaments").

La loi de 2004 met en place une nouvelle institution, l'Agence de la biomédecine, destinée à encadrer notamment les activités de recherche, la greffe, la reproduction, l'embryologie et la génétique humaines. Établissement public administratif de l'État, placée auprès du ministre de la Santé, elle a notamment pour mission : de participer à l'élaboration et à l'application de la réglementation et des règles de bonne pratique ; de suivre, d'évaluer et de contrôler les activités médicales et biologiques relevant de sa compétence ; d'agréer les praticiens et les protocoles de recherche. L'État (et ses experts) réaffirme ainsi sa forte présence dans le champ de la bioéthique à travers l'ensemble d'instances sous son contrôle: CCNE, Conseil d'État, Agence de la biomédecine et Office parlementaire d'évaluation des choix scientifiques et technologiques, États généraux de la bioéthique, etc.

La révision de 2011 : beaucoup de bruit pour rien...

Pour assurer l'adaptation du droit aux évolutions de la science, il était prévu que la loi de bioéthique du 6 août 2004 (issue de la première révision des lois de 1994) soit réexaminée dans un délai de cinq ans après son entrée en vigueur. Ce nouveau processus de révision législative s'est caractérisé par l'intervention de plusieurs instances officielles qui ont préparé la rédaction du projet de loi. La Commission nationale consultative des droits de l'Homme $(\mathrm{CNCDH})$ fut la première à rendre un avis général afin de rappeler les principes sur lesquels se fonde le dispositif bioéthique français ${ }^{18}$. Le 9 octobre 2008, le Comité consultatif national d'éthique $(\mathrm{CCNE})$ a rendu public un mémoire ${ }^{19}$, qui laisse la porte ouverte à l'évolution de certaines restrictions, comme l'anonymat des donneurs de gamètes. Concomitamment au CCNE, l'Agence de la biomédecine publie un bilan d'application de la loi et propose quelques pistes de travail pour sa révision ${ }^{20}$. L'Office parlementaire d'évaluation des choix scientifiques et technologiques (OPECST) présente à son tour le rapport sur l'évaluation de la loi de 2004, fruit de trois auditions publiques associant chercheurs, juristes, sociologues, philosophes et membres d'instances de la biomédecine et de comités d'éthique ${ }^{21}$. L'OPECST élargit sa réflexion en englobant les neurosciences et l'imagerie cérébrale. Appelé par le gouvernement à donner son avis, le Conseil d'État a constitué un groupe de travail en vue de procéder à une évaluation de la loi et d'établir des préconisations ${ }^{22}$. L'avis du Conseil d'État exercera une forte influence sur le contenu de la révision.

\footnotetext{
18 http:// www.cncdh.fr/article.php3?id article=493 [lien consulté le 21 avril 2011].

19 «Questionnement pour les États généraux de la bioéthique », CCNE, 9 octobre 2008, n 105.

20 « Bilan d'application de la loi de bioéthique du 6 août 2004 ». Rapport à la ministre de la Santé, de la J eunesse, des Sports et de la Vie associative, octobre 2008.

21 http:/ / www.assemblee-nationale.fr/ 13/rap-off/i1325-tI.asp

${ }^{22}$ Conseil d'État, La révision des lois de bioéthique, Études du Conseil d'État, Paris, La Documentation française, 2009.
} 
Daniel Borrillo, «La République des experts dans la construction des lois : le cas de la bioéthique », Histoire@Politique. Politique, culture, société, nº 14, mai-août 2011, www.histoire-politique.fr

Les États généraux de la bioéthique, organisés par le président de la République et la ministre de la Santé, constituent une innovation dans le débat bioéthique. Présentée par les autorités comme un vaste débat citoyen ayant comme objectif d'informer, de discuter et de dégager des propositions, cette "consultation nationale" a consisté en la réunion de trois jurys de seize personnes formées par des experts et très encadrée par un comité de pilotage particulièrement conservateur. L'extrême prudence du rapport des États généraux ${ }^{23}$ apparaît comme le résultat du bon sens populaire et permet de cautionner l'immobilisme souhaité par le gouvernement. C'est pourquoi, les milliers d'avis d'internautes et les deux cent douze rencontres qui ont rythmé cinq mois de débat ne sont pas pris en compte dans le rapport. Alain Graf, rapporteur général des états généraux, s'explique comme suit : « Parce qu'ils n'ont pas été sélectionnés selon des critères de représentativité, les témoignages individuels diffusés sur le site des États généraux de la bioéthique ne sauraient être appréhendés de la même manière que les contributions issues d'un débat et d'une réflexion collective » (p. 7). « Les avis publiés sur le site requièrent certaines précautions de lecture [...], l'analyse des avis déposés fait plutôt apparaître des positions figées [...], ils s'apparentent le plus souvent à l'expression d'un jugement isolé...» (p. 55). Après avoir auditionné plus d'une centaine d'experts, la Mission parlementaire d'information sur la révision des lois de bioéthique (dernière étape avant le débat au Parlement) adopte quatre-vingtquatorze propositions qui ne changent pas la structure générale des lois antérieures ${ }^{24}$. Malgré le nombre d'auditions, rapports et forums qui ont précédé le travail de la Mission parlementaire, celle-ci a opté pour une révision a minima, suivant les propositions du Conseil d'État et écartant les avancées suggérées par les autres instances consultatives, les sociétés savantes et les contributions de la société civile.

\section{Les principes sur lesquels repose la bioéthique en France}

Les articles 16 à 16-9 du Code civil posent les principes fondamentaux de respect du corps humain qui gouvernent les pratiques biomédicales, à des fins thérapeutiques comme à des fins scientifiques. Pour l'essentiel, ces principes sont issus de la loi n 94-653 du 29 juillet 1994, complétée par la réforme de 2004, en particulier, pour poser explicitement l'interdiction du clonage. Sont ainsi consacrés : « le respect de l'être humain dès le commencement de sa vie »; l'inviolabilité du corps humain; l'extrapatrimonialité du corps humain, de ses éléments et de ses produits; la nonatteinte à l'intégrité du corps humain, sauf «nécessité médicale (...) ou à titre exceptionnel dans l'intérêt thérapeutique d'autrui » ; la sauvegarde de l'intégrité de l'espèce humaine qui conduit à prohiber les pratiques eugéniques ; l'interdiction des transformations des caractères génétiques d'une personne dans le but de modifier sa descendance ; la gratuité des expérimentations sur la personne, du prélèvement et de

\footnotetext{
${ }^{23} \mathrm{http}$ :// www.etatsgenerauxdelabioethique.fr/ uploads/rapport final.pdf [lien consulté le 19 avril 2011].

${ }^{24}$ Assemblée nationale, « Rapport d'information de la mission d'information sur la révision des lois de bioéthique », 20 janvier 2010 (Rapporteur J ean Leonetti).
} 
Daniel Borrillo, «La République des experts dans la construction des lois : le cas de la bioéthique », Histoire@Politique. Politique, culture, société, nº 14, mai-août 2011, www.histoire-politique.fr

la collecte d'éléments et produits du corps humain; l'interdiction des conventions « portant sur la procréation ou la gestation pour le compte d'autrui » ; l'anonymat entre donneur et receveur d'éléments ou produits du corps. Enfin, l'accès à l'AMP est réservé aux couples hétérosexuels stériles. La dernière révision des lois reconduit ces mêmes principes. Présentés comme universels et apolitiques, ces principes, lorsqu'ils s'appliquent de manière réflexive (c'est-à-dire envers soi-même), suscitent un certain nombre de paradoxes que le discours des experts tente de dissiper en particulier celui d'interdire la libre disposition du corps et de la vie par l'individu lui-même.

\section{La controverse bioéthique : l'expertise en Humanité contre les droits individuels}

La recherche d'éléments permettant de tracer les frontières du permis et de l'interdit en matière de recherche médicale constitue le socle de la réflexion bioéthique. Le nombre et la complexité des théories ne nous permettent pas de rendre compte, même de manière abrégée, des principales conceptions philosophiques ${ }^{25}$. Nous nous limiterons aux deux grands pôles, selon lesquels l'origine et la fin de la norme bioéthique sont à chercher dans la société elle-même, à travers un processus de délibération argumentative (immanence) ou au-dessus d'elle-même en se référant à un ordre extérieur s'imposant aux individus (transcendance ou métaphysique) ${ }^{26}$. Pour cette dernière conception, la norme est universelle, invariable et antérieure au politique (essentialisme) tandis que, pour le courant immanent, la norme apparaît comme une variable dépendante de la société et de l'histoire (constructivisme) ${ }^{27}$. La distinction entre morale personnelle et sphère du politique constitue un autre élément capital de la conception immanente de type libéral. Pour les uns, le libre choix personnel doit primer tandis que, pour les autres, l'État peut et doit gouverner la vie des individus, y compris dans sa dimension la plus intime. La sacralité et la non-disponibilité de la vie constituent le soubassement de la pensée métaphysique. L'orientation opposée se fonde sur la qualité et la disponibilité pour l'individu de sa propre vie.

En France, un courant idéologique - néo-traditionaliste - allie un contenu essentialiste à une rhétorique apparemment laïque et ceci afin de condamner, ou tout simplement de soustraire de la délibération politique, certaines pratiques telles la gestation pour autrui, l'AMP pour les célibataires, l'homoparentalité ou l'euthanasie, considérées comme des « agissements » susceptibles de porter atteinte aux principes immuables comme l'ordre symbolique, le fondement anthropologique du droit, la dignité humaine...

\footnotetext{
25 Traditionnellement, on appelle théories déontologiques ou antiperfectionnistes celles qui donnent la priorité au juste et théories téléologiques ou perfectionnistes celles qui placent le bien au-dessous du juste.

${ }^{26}$ La complexité du débat et son étendue dans le temps témoignent du caractère permanent de ce type de controverse à laquelle selon Walzer nous sommes condamnés ad infinitum (M. Walzer, «The Communitarian Critique of Liberalism », Political Theory, n 18, p. 6-23).

${ }_{27}$ Pour une analyse plus approfondie des différentes lignes de partages du débat bioéthique en France, voir Dominique Mehl, Naître? La controverse bioéthique, Paris, Bayard, 1999.
} 
Daniel Borrillo, « La République des experts dans la construction des lois : le cas de la bioéthique », Histoire@Politique. Politique, culture, société, nº 14, mai-août 2011, www.histoire-politique.fr

\section{Les constructivistes et l'éthique immanente}

Pour la pensée immanente, la société accomplit son histoire et la règle morale trouve sa source à l'intérieur d'elle-même. L'autonomie et le consentement constituent les piliers de cette « éthique ». La liberté est composée de deux éléments indissociables : le droit subjectif de l'individu en tant que faculté d'agir selon ses propres choix et la créance sur tous les autres membres de la société qui doivent s'abstenir d'intervenir dans cette sphère de la vie privée. La seule limite à la liberté est le préjudice à autrui, sans lequel tout acte doit être considéré comme légitime. De même, ce n'est pas la soumission à une règle extérieure présentée comme universelle et naturelle (éthique de la conviction) qui détermine la moralité d'un acte mais l'attention aux conséquences concrètes d'une action (éthique de la responsabilité). La fonction du droit consiste à respecter la liberté accordée à chacun de poursuivre ses propres objectifs, mais dans le respect des droits de l'ensemble des citoyens. Il s'agit d'une conception fondée sur la modernité en tant que possibilité politique réflexive de changer les règles du jeu de la vie sociale. La morale étant de nature relative, chaque société peut se donner de manière autonome ses propres règles de comportement. Pour l'éthique de la responsabilité, la recherche du bonheur individuel constitue également une règle herméneutique. Connue sous le terme « utilitarisme», cette philosophie peut être résumée dans l'expression du principe d'utilité visant la «maximisation du plus grand bonheur du plus grand nombre». Philosophiquement parlant, le sens du terme « utilitariste » ne correspond nullement à son acception courante, c'est-à-dire ce qui est profitable ou avantageux, mais désigne ce qui contribue à maximiser le bien-être d'une population. En ce sens, l'utilitarisme s'oppose à l'individualisme puisqu'il cherche l'extension du bonheur au plus grand nombre. Le principe directeur de cette éthique est celui de la justice distributive : il faut satisfaire le plus possible d'intérêts du plus grand nombre de personnes, quels qu'ils soient. La recherche du bonheur et l'absence de souffrance des tiers constituent les principaux éléments déterminant la moralité d'un acte.

D'un point de vue pratique, la démarche utilitariste consiste à mettre en relation le positif d'une action en fonction de l'utilité rationnelle de ses conséquences. Ainsi, une action sera jugée moralement acceptable à partir du moment où elle est plus utile que nuisible, tant du point de vue de la personne concernée que vis-à-vis du bien commun. Fondée sur l'autonomie, en tant que faculté personnelle de s'autodéterminer, cette conception renforce la responsabilisation des individus et des sociétés en obligeant l'acteur social à mesurer systématiquement les conséquences concrètes de ses actes. Dépourvu d'une règle universelle et d'une hiérarchie des valeurs, le courant non essentialiste estime d'une part que l'autorité ne peut agir sur la conviction mais uniquement sur l'intérêt et, d'autre part, que tous les intérêts se valent et doivent être pris en considération d'égale manière. De surcroit, les actes ne sont pas jugés en eux-mêmes (essence) mais en fonction du contexte dans lequel ils se déploient et selon les conséquences réelles qu'ils produisent. 
Daniel Borrillo, «La République des experts dans la construction des lois : le cas de la bioéthique», Histoire@Politique. Politique, culture, société, nº 14, mai-août 2011, www.histoire-politique.fr

La moralité d'une action est considérée comme une réalité matérielle qui peut être démontrée et mesurée, à partir des motivations élémentaires des êtres vivants sensibles. Dans ce but, la bioéthique non métaphysique introduit la notion de bilan éthique. À partir d'une analyse permettant de mesurer le coût et le bénéfice d'une action, il est possible d'autoriser certaines interventions, mêmes irréversibles, consenties par les patients. Ainsi, le citoyen en fin de vie devient le centre du dispositif légal, afin de lui permettre la réalisation de sa dernière liberté. Dans une République laïque, c'est à l'individu et non pas à l'État de décider, sous certaines conditions, quand et comment mettre fin à ses jours ${ }^{28}$. De même, lorsqu'une femme dispose d'ovocytes sains mais ne peut pas pour des raisons médicales porter l'enfant, il est éthiquement valable qu'elle puisse recourir à la gestation pour autrui (à condition qu'il n'y ait pas exploitation et que tous les protagonistes de cette entreprise soient consentants).

Robert Badinter a su incarner ce courant avec brio pendant les premières années du débat bioéthique. Selon lui, « de sa vie privée, tout adulte est maître absolument dans notre société » et «parmi les droits que l'être humain doit se voir reconnaître et garantir par la loi pour pouvoir espérer atteindre l'épanouissement de sa personne qui est la fin de toute l'organisation sociale dans une démocratie, le droit de donner la vie, de remplir plus concrètement sa destinée humaine et d'assurer sa descendance nous paraît un droit essentiel. Le droit de donner la vie ne peut être refusé à une femme qui veut avoir un enfant. On ne saurait donc lui en interdire les moyens ${ }^{29}$ ».

Pendant les auditions pour la dernière révision des lois de bioéthique, des arguments immanents ont été avancés pour dénoncer la pénalisation des mères porteuses, obligeant ainsi les femmes françaises incapables de mener à bien une grossesse à se rendre à l'étranger pour réaliser leur projet parental. Face à l'idée essentialiste selon laquelle toute maternité implique un lien profond et intime avec le foetus, la philosophe Elisabeth Badinter a souligné que, dans la pratique, « on peut porter un enfant sans faire de projet, sans fantasmer, sans tricoter une relation avec lui ${ }^{30} »$. De même, face au refus opposé à la gestation pour autrui au nom de la nécessaire marchandisation du corps, les travaux sociologiques de Dominique Mehl ont permis de montrer que «la motivation financière des gestatrices n'est pas la motivation première $^{31}$ ». Autrement dit, rien ne justifie d'interdire à une femme la réalisation d'un geste altruiste comme celui de porter l'enfant de sa sœur incapable de mener à bien une gestation pour des raisons médicales, par exemple. Toute en désapprouvant cette pratique, la politologue Monique Canto-Sperber considère que, lorsque les personnes sont consentantes, la loi n'a pas à dire ce qu'elles doivent faire d'ellesmêmes et de leurs corps alors que nul ne serait lésé 32 .

28 «Liberté, Égalité, Fraternité. Une loi de Liberté pour tous les Français », Audition de Jean-Luc Roméro, président de l'Association pour le droit de mourir dans la dignité, à l'Assemblé nationale, le 4 juin 2008.

29 « Les droits de l'homme face aux progrès de la médecine, de la biologie et de la biochimie », Le Débat, $\mathrm{n}^{\circ} 36$, septembre 1985.

30 Alain Claeys et J ean-Sébastien Vialatte, « Rapport sur l'évaluation de l'application de la loi $n^{\circ} 2004$ 800 du 6 août 2004 relative à la bioéthique », 2008.

${ }^{31}$ Audition de Dominique Mehl, sociologue au CNRS, op. cit., p. 146.

32 Mission d'information sur la révision des lois bioéthiques, 4 mars 2009. 
Daniel Borrillo, «La République des experts dans la construction des lois : le cas de la bioéthique », Histoire@Politique. Politique, culture, société, nº 14, mai-août 2011, www.histoire-politique.fr

Les représentants du courant constructiviste, bien que provenant d'horizons différents, peuvent tous faire leur la formule de Benjamin Constant: "Prions l'autorité de rester dans ses limites; qu'elle se borne à être juste, nous nous chargerons d'être heureux ${ }^{33}$. »

\section{L'expertise essentialiste et l'éthique transcendante}

Basé sur la croyance en une vérité absolue, le courant métaphysique considère que les lois humaines trouvent leur fondement dans des forces extérieures et supérieures qui les déterminent ${ }^{34}$. La société doit s'abandonner à ces principes immuables car « la loi naturelle est universelle dans ses prescriptions et son autorité s'étend à tous les hommes ${ }^{35}$ ». Pour l'Église, « ces préceptes positifs, qui prescrivent d'accomplir certaines actions et de cultiver certaines attitudes, obligent universellement et ils sont immuables ; ils réunissent dans le même bien commun tous les hommes de toutes les époques de l'histoire ${ }^{36} »$. Selon la pensée métaphysique, avec la modernité disparaît toute référence à des valeurs communes et à une vérité absolue pour tous : " La vie sociale s'aventure dans les sables mouvants d'un relativisme absolu et tout est matière à convention, tout est négociable ${ }^{37} . . . »$ Ce n'est donc pas la délibération démocratique mais la contemplation des lois éternelles qui détermine la frontière du permis et de l'interdit en matière bioéthique. Le religieux apparaît comme la forme paradigmatique du courant métaphysique. La tradition se substitue à l'argumentation à l'heure de trouver la légitimation de la norme. Déterminé par une éthique des convictions conçue comme fidélité à des valeurs, le courant métaphysique se soucie peu des conséquences concrètes d'une action. Le catéchisme de l'Église catholique dispose dans son canon 1756 qu'il « est erroné de juger de la moralité des actes humains en ne considérant que l'intention qui les inspire, ou les circonstances (milieu, pression sociale, contrainte ou nécessité d'agir, etc.) qui en sont le cadre. Il y a des actes qui par eux-mêmes et en eux-mêmes, indépendamment des circonstances et des intentions, sont toujours gravement illicites en raison de leur objet...».

Peu importe les effets d'une décision sur la vie réelle pourvu qu'elle soit en conformité avec ces valeurs absolues. Ainsi, pour l'Église, « l'utilisation des embryons ou des foetus humains comme objets d'expérimentation constitue un crime contre leur dignité d'êtres humains, qui ont droit à un respect égal à celui dû à l'enfant déjà né et à toute personne ${ }^{38} »$. Même avec une malformation le rendant inviable ou indépendamment qu'il soit le fruit d'un viol, l'avortement est condamné de manière absolue. Selon l'Église, « l'être humain doit être respecté - comme une personnedès le premier instant de son existence ${ }^{39} »$. Et, lorsque l'évêque de Recife (Brésil)

\footnotetext{
33 De la liberté des anciens comparée à celle des modernes, 1819.

34 « La loi morale est l'œuvre de la Sagesse divine » (Catéchisme de l'église catholique canon 1950).

$35 \mathrm{~J}$ ean-Paul II, Encyclique Veritatis splendor 51(3).

36 Encyclique citée, 52.

${ }^{37} \mathrm{~J}$ ean-Paul II, Encyclique Evangelium vitae 20.

38 J ean-Paul II, Encyclique Evangelium vitæ, n. 63 : AAS 87 (1995), 472-473, La Documentation catholique 92 (1995), p. 383.

39 J ean-Paul II, Donum Vitae. Instruction romaine de la congrégation pour la doctrine de la foi sur "Le respect de la vie humaine naissante et la dignité de la procréation", 22 février 2007.
} 
Daniel Borrillo, «La République des experts dans la construction des lois : le cas de la bioéthique », Histoire@Politique. Politique, culture, société, nº 14, mai-août 2011, www.histoire-politique.fr

excommunie en 2009 l'équipe médicale qui avait pratiqué une interruption de grossesse sur une mineure de neuf ans violée par son beau-père et enceinte de jumeaux, il applique les principes de la foi catholique. Peu importe la vie de la fillette (elle mesurait $1,36 \mathrm{~m}$ et pesait $33 \mathrm{~kg}$ ), incapable de toute évidence de mener cette grossesse à terme ${ }^{40}$.

Les religions condamnent le clonage thérapeutique, même si cette condamnation risque d'entraver la recherche scientifique et l'action thérapeutique, l'homme n'ayant pas le droit de créer le vivant même si cela permet de sauver des vies. Aussi, pour l'Église, le don d'organe doit être un acte d'amour totalement gratuit puisque «le corps de chaque personne, avec l'esprit qui est donné de façon singulière à chacun, constitue une unité indissociable sur laquelle est inscrite l'image de Dieu même ${ }^{41}$ ». De même, pour l'Islam, « tout organe humain prélevé chez un vivant ou un cadavre ne peut jamais faire l'objet d'une vente car cet organe provient d'un corps qui n'appartient pas à l'homme mais à Dieu qui le lui a confié (amanah) ${ }^{42} »$. Peu importe que la gratuité absolue aboutisse à une pénurie d'organes ${ }^{43}$, seule compte l'obéissance à la prescription divine. Le lobbying de l'Église catholique en la matière est affiché sans complexes et, par le biais des documents officiels, elle entend influencer directement le législateur français ${ }^{44}$.

En matière de procréation, aussi bien l'Islam, le Judaïsme que l'Église de Rome considèrent que l'accord avec l'ordre naturel rend uniquement licite certaines techniques extrêmement limitées. Ainsi, «l'insémination artificielle homologue à l'intérieur du mariage ne peut être admise, sauf dans le cas où le moyen technique ne se substitue pas à l'acte conjugal, mais apparaît comme une facilité et une aide afin que celui-ci rejoigne sa fin naturelle ${ }^{45} \gg$. Par ailleurs, le mariage et la famille constituent pour l'Église «le contexte authentique où la vie humaine trouve son origine. En son sein, la vie provient d'un acte qui exprime l'amour réciproque entre l'homme et la femme. Une procréation réellement responsable vis-à-vis de l'enfant qui va naître doit être le fruit du mariage ${ }^{46} »$, c'est pourquoi ni les femmes célibataires ni les couples homosexuels ne doivent accéder aux techniques de procréation artificielle. Pour la jurisprudence islamique, « le mariage étant un contrat entre un homme et une femme, tout au long de leur union il ne sera pas admis qu'une troisième personne interfère dans leur vie sexuelle ou contribue à la procréation d'un de leurs enfants en fournissant des gamètes, un œuf, un embryon ou un utérus. Dans

\footnotetext{
40 « L'avortement d'une fillette de 9 ans bouleverse le Brésil », Le Figaro, 9 mars 2009.

${ }^{41}$ Discours du pape Benoît XVI aux participants au congrès international sur le thème du don d'organes organisé par l'académie pontificale pour la vie, le vendredi 7 novembre 2008.

42 «Bioéthique et Jurisprudence islamique », http:// www.fm.usj.edu.lb/ anciens/files/ bioethique/islam.pdf, p. 10 .

43 En France par exemple sur 10000 personnes en attente de greffe de rein, seulement un tiers d'entre elles ont pu être transplantées en 2008.

44 Voir Mgr Pierre d'Ornellas (dir.), Bioéthique. Propos pour un dialogue, Lethielleux, coll. « Documents d'Église », 2009.

45 Congrégation pour la doctrine de la foi, Instruction Donum vitae, II, B, 6 : 1. c., 94, La Documentation catholique 84 (1987), p. 358.

${ }^{46}$ Congrégation pour la doctrine de la foi, Instruction Donum vitae, I, 1: AAS 80 (1988), 78-79 ; La Documentation catholique (1987), p. 355.
} 
Daniel Borrillo, «La République des experts dans la construction des lois : le cas de la bioéthique », Histoire@Politique. Politique, culture, société, nº 14, mai-août 2011, www.histoire-politique.fr

un couple où l'homme est totalement et définitivement infertile, la stérilité doit être acceptée et aucun traitement ne devrait être tenté ou entrepris ${ }^{47}$ ».

Le magistère de l'Église de Rome considère le diagnostic préimplantatoire comme « l'expression de cette mentalité eugénique qui accepte l'avortement sélectif pour empêcher la naissance d'enfants affectés de différents types d'anomalies. Une pareille mentalité est ignominieuse et toujours répréhensible, parce qu'elle prétend mesurer la valeur d'une vie humaine seulement selon des paramètres de "normalité" et de bien-être physique, ouvrant ainsi la voie à la légitimation de l'infanticide et de l'euthanasie $\mathrm{e}^{48}$ ». La députée Christine Boutin incarne en France le combat du courant métaphysique et plus particulièrement celui de la doctrine catholique. Dès les premiers rapports, cette députée n'a cessé de militer contre certains contenus des lois bioéthiques, considérées trop laxistes et qui font de l'embryon « un otage et un esclave ».

Un autre aspect de la transcendance réside dans l'idée selon laquelle la science pourrait, par son propre fonctionnement, non seulement s'autolimiter mais fournir la solution aux problèmes posés par son propre développement. La science se substituerait ainsi à la politique dans la recherche des réponses aux problèmes éthiques. Il suffirait, pour ce faire, d'appliquer correctement ses principes et sa méthode. Cette conception, dénommée scientisme, n'a été ouvertement soutenue par aucun acteur social dans la délibération bioéthique en France et, à part l'économiste Bertrand Lemennicier ${ }^{49}$, personne n'a assumé une défense radicale de la liberté scientifique conjuguée avec la liberté du marché en tant que forme légitime de régulation des sciences de la vie et de la santé.

\section{La figure de l'expert néo-traditionaliste}

Comme le courant métaphysique, certains auteurs utilisent des arguments essentialistes afin d'extraire de la politique un certain nombre de questions bioéthiques considérées comme non négociables. Si le conservatisme est, selon l'expression de Karl Mannheim, le «traditionalisme devenu conscient », le néoconservatisme est une forme de traditionalisme inconscient; une sorte de mélange entre un contenu conservateur et un continent apparemment neutre, prenant souvent la forme de l'anthropologie ou de la psychanalyse. Comme les courants religieux, la pensée néo-conservatrice est attachée à démontrer et à dénoncer les abus de la science, le «poison » de l'individualisme, la marchandisation de l'humain et la désintégration sociale produite par la modernité50. Au début du débat bioéthique, le juriste Bernard Edelman apparait comme l'un des avocats les plus brillants de cette conception philosophique: " Nous sommes entrés dans l'ère de l'artifice, nous

\footnotetext{
${ }^{47}$ Op. cit., p. 17.

48 Congrégation pour la doctrine de la foi, op. cit., paragraphe 22.

49 Bertrand Lemennicier, « Le corps humain : propriété de l'État ou propriété de soi ? », Droits, $n^{\circ} 13$, 1991.

50 Irène Théry n’hésitait pas à dénoncer le droit devenu « un outil de gestion des désirs privés ». Cité par le «Comité Protestant évangélique pour la Dignité humaine» pour s'opposer à la revendication homosexuelle.
} 
Daniel Borrillo, «La République des experts dans la construction des lois: le cas de la bioéthique », Histoire@Politique. Politique, culture, société, n 14, mai-août 2011, www.histoire-politique.fr

fabriquons tout: des plantes, des animaux, des mémoires, des intelligences; nous inventons tout (...). L'Occident a réalisé son antique fantasme de maîtrise de la nature, et la nature maîtrisée a perdu son sens ${ }^{51}$...»

Obsédée par la perte des repères et par les risques d'effacement de l'ordre traditionnel, la pensée néo-traditionaliste se fait gardienne de l'ordre établi, présenté comme une structure anthropologique immuable. Alors que l'idéologie conservatrice invoque ouvertement l'ordre naturel, les néo-conservateurs - issus aussi bien du monde ecclésiastique ${ }^{52}$ que de l'univers académique - font souvent appel aux sciences humaines pour justifier leurs proscriptions ${ }^{53}$. La dimension descriptive des sciences sociales est ouvertement écartée et, par un abus épistémologique, ces sciences sont invoquées en tant que savoir imposant aux individus et aux sociétés des règles invariantes auxquelles ils doivent se soumettre sous peine de mettre en danger l'humain ${ }^{54}$. La notion de dignité humaine appliquée à la relation réflexive de l'individu à lui-même et la prétendue fonction anthropologique du droit constituent l'appareil idéologique permettant de limiter la liberté au nom d'un principe impératif s'imposant à tous les humains en tant que détenteurs d'une portion d'humanité à laquelle il faut se subordonner. Comme le note Olivier Cayla, " contrairement à la perspective moderne où, par nature, l'individu s'appartient (...), la logique de la dignité posture que, par nature, l'individu ne s'appartient pas, mais appartient à sa nature qui le transcende ${ }^{55} »$.

\footnotetext{
51 Bernard Edelman, « Vers une approche juridique du vivant », dans L'homme, la nature et le Droit, Paris, Christian Bourgois, 1988. Cette ouvrage collectif d'inspiration heideggérienne n'a pas eu d'influence sur l'élaboration de la loi mais a séduit les journalistes et l'opinion publique.

52 Dans son encyclique Veritatis splendor, J ean-Paul II rappelle les propositions de l'Église quant à l'utilisation d'un langage modernisé : « Le Concile Vatican II a invité les théologiens, "à chercher la manière toujours plus adaptée pour communiquer la doctrine aux hommes de leur temps " (...) Les efforts de nombreux théologiens, soutenus par les encouragements du Concile, ont déjà porté leurs fruits, par des réflexions intéressantes et utiles sur les vérités de la foi qu'il faut croire et appliquer dans la vie, présentées sous des formes qui répondent davantage à la sensibilité et aux interrogations des hommes de notre temps » (paragraphe 29).

53 Suivant cette recommandation, de nombreux théologiens ne parlent plus d'ordre naturel mais d'anthropologie et de psychanalyse. Voir en ce sens, Xavier Thévenot, Homosexualités masculines et morale chrétienne (Paris, Cerf, 1992) et Xavier Lacroix, L'amour du semblable. Questions sur l'homosexualité, Paris, Cerf, 1996).

54 Dans un courrier adressé à Claude Lévi-Strauss le 13 juillet 1999 (durant le débat sur le PaCS), Éric Fassin s'inquiétait, en voyant citer le nom du père de l'anthropologie structurale (pourtant demeuré silencieux dans ce débat public), du «statut de la référence scientifique invoquée de manière abusive comme expertise » par les savants non moins que par les politiques. Dans une lettre du 19 juillet 1999, Claude Lévi-Strauss répond à la lettre d'Eric Fassin comme suit : " L'éventail des cultures humaines est si large, si varié (et d'une manipulation si aisée) qu'on y trouve sans peine des arguments à l'appui de n'importe quelle thèse. Cette familiarité acquise avec les usages les plus divers lui enseigne - au mieux une certaine sagesse qui peut n'être pas inutile à ses contemporains; sans oublier toutefois que les choix de société n'appartiennent pas au savant en tant que tel, mais - et lui-même en est un - au citoyen », dans Daniel Borrillo, Éric Fassin, Au-delà du PaCS, Paris, PUF, 2e édition 1999, p. 110.

55 Olivier Cayla et Yan Thomas, Du droit de ne pas naître. À propos de l'affaire Perruche, Paris, Gallimard, 2002, p. 50.
} 
Daniel Borrillo, «La République des experts dans la construction des lois : le cas de la bioéthique », Histoire@Politique. Politique, culture, société, nº 14, mai-août 2011, www.histoire-politique.fr

Ces « gestionnaires du sacré », pour reprendre l'expression de Dominique Memmi ${ }^{56}$, sont devenus les nouveaux surveillants de nos corps et de nos conduites corporelles. Ainsi, lors des auditions publiques à l'Assemblée nationale, une juriste a commencé son intervention en soulignant que « le législateur ne peut pas ne pas tenir compte des structures plus dures, plus profondes et anciennes, et des catégories juridiques à l'intérieur desquelles les règles prennent place, et qui leur donnent sens ${ }^{57} »$. Parce qu'il est « essentiel pour ne pas devenir fou » ou puisque «la nature l'impose », la juriste considère qu'«il n'est pas opportun d'ajouter encore de l'instabilité et de la perte de repères sur cet élément fondamental de détermination du père et de la mère ». Elle s'est dite, par conséquent, « opposée à toute modification des critères de la maternité». Et si la vulgate anthropologique ne suffisait pas à convaincre les députés, l'appel au sens commun vient appuyer ses arguments : " J e considère que le bon sens populaire s'opposerait complètement à ce que l'on consacre une maternité génétique qui ne soit pas gestationnelle. J 'estime qu'en la matière, l'expérience de la totalité des individus ne doit pas être bafouée au point que les femmes et les mères ne se reconnaîtraient plus dans leurs fonctions et nature ${ }^{58}$. »Contrairement à la doctrine des civilistes de l'époque ${ }^{59}$, cette ancienne membre du comité d'éthique avait déjà milité ardemment pour l'adoption d'un dispositif bioéthique contraignant : « Il y a urgence si l'on veut éviter que par effet de banalisation, le développement rapide et incontrôlé de ces techniques n'introduise dans l'ensemble des représentations culturelles l'idée que la reproduction humaine ne se différencie plus de ce que les hommes ont fait de la reproduction animale et qu'elle obéit, de fait, aux lois économiques d'une production industrialisée, commercialisée et programmée, de $l^{\prime}$ homme ${ }^{60}$. » Utilisant l'argument de la pente fatale (conduisant mécaniquement du tolérable à l'inacceptable) ${ }^{61}$, une autre juriste auditionnée par la Mission parlementaire met en garde les députés contre les risques irréversibles qu'ils peuvent provoquer s'ils « enlèvent les bornes » ou si le législateur « dilue les repères du droit de la famille», lequel «structure et donne du sens à la filiation». En tant que gardienne de la dignité humaine, l'experte n'hésite pas à dénoncer les pratiques des mères porteuses, considérées comme de « l'esclavage procréatif 62 ».

Les conservateurs pensent que la cohérence et la lisibilité du droit de la filiation passent principalement par la subordination de la loi au biologique, surtout lorsque la revendication des couples homosexuels s'inscrit dans l'ordre du jour politique. Aussi,

\footnotetext{
56 Dominique Memmi, Les Gardiens du corps. Dix ans de magistère bioéthique, Paris, Éditions de l'École des hautes études en sciences sociales, 1996.

${ }^{57}$ Catherine Labrusse-Riou, « Rapport sur l'évaluation de l'application de la loi du 6 août 2004 relative à la bioéthique par M.M. les députés Alain Claeys et J ean-Sébastien Vialatte », n 1325, tome 2, Auditions, décembre 2008, p. 61-69.

58 Idem.

59 En particulier François Terré et J ean Carbonnier.

60 Jean-Louis Baudouin et Catherine Labrusse-Riou, Produire l'homme: de quel droit?, Paris, PUF, 1987.

${ }^{61}$ Pour une analyse approfondie de l'argument de la « pente fatale », voir Ruwen Ogien, La Vie, la Mort, l'État. Le débat bioéthique, Paris, Grasset, 2009.

62 Audition de $\mathrm{M}^{\mathrm{me}}$ Françoise Dekeuwer-Défossez, Mission d'information sur la révision des lois bioéthiques, 18 mars 2009, retransmission en différé (site del'Assemblée nationale).
} 
Daniel Borrillo, «La République des experts dans la construction des lois : le cas de la bioéthique», Histoire@Politique. Politique, culture, société, nº 14, mai-août 2011, www.histoire-politique.fr

pour le doyen de la faculté de théologie de Lyon, «la cohérence de la filiation doit donc être un critère et une préoccupation. Que l'enfant naisse de l'union de deux corps, ou même de deux cellules, masculine et féminine, est chargé de sens et de valeur. Puisque ce sont des liens qui sont en jeu, ils sont nécessairement institués, on ne peut pas s'en tenir au principe anglo-saxon du libre choix individuel. Le lien social passe par la sauvegarde d'un socle anthropologique commun ${ }^{63}$ ». Face à « l'individualisme effréné » et à la décadence du droit moderne de la famille, l'une des juristes auditionnées avait déjà prêché en faveur d'un « droit du principe » fondé sur «l'ordre symbolique de la différence des sexes», car, selon elle, "c'est la structure même de la société qui serait menacée par des familles trop anormales, par des filiations trop atypiques. Il n'est pas socialement supportable [...] que des couples homosexuels soient féconds. Cela risquerait de déstabiliser complètement les relations familiales et sociales et cela pourrait à terme nuire à la structuration psychologique de tous les enfants, même nés dans des familles normales ${ }^{64} »^{\prime}$. Le préjugé est ainsi assumé ouvertement dans l'expertise familiale en tant que principal argument contre les familles homoparentales ${ }^{65}$.

La naturalisation des liens de filiation, l'ordre symbolique de la différence des sexes $^{66}$, la valence différentielle des sexes ${ }^{67}$ ou la loi d'Edipe constituent des formes réactualisées de la transcendance d'autant plus efficaces qu'elles ne font pas appel à la religion pour justifier leur prosélytisme mais utilisent la vulgate anthropologique et psychanalytique ${ }^{68}$, plus compatibles avec l'exigence de laïcité. Cette nouvelle figure du dogmatisme a pour objectif d'extraire du politique un certain nombre de questions comme l'homoparentalité, la gestation pour autrui, l'insémination post mortem, le droit de mourir dans la dignité... de telle sorte que toute revendication fondée sur les principes du droit (égalité, non-discrimination, droit à la procréation, libre disposition de son corps...) soit automatiquement refusée au nom des impératifs prépolitiques, considérés comme constitutifs de la culture ${ }^{69}$. Comme le note Olivier Cayla, la stratégie de la pensée néo-conservatrice consiste à « opposer aux prétentions subjectives de l'individu les bornes d'un ordre impératif et transcendant, dont le contenu normatif n'est connaissable que de ses seuls interprètes autoproclamés ${ }^{70} »$.

\footnotetext{
63 Xavier Lacroix, « De la bioéthique à l'anthropologie », Blog Bioéthique de la Conférence des évêques de France : http:// www.bioethique.catholique.fr [lien consulté le 21 avril 2011].

64 Françoise Dekeuwer-Défossez, « Réflexions sur les mythes fondateurs du droit contemporain de la famille », RTD civil, 1995.

65 Pour une analyse approfondie de la question, voir Daniel Borrillo et Pierre Lascoumes, Amours égales ? Le Pacs, les homosexuels et la gauche, Paris, La Découverte, coll. « Sur le vif », Paris, 2002.

66 Terme vulgarisé par Irène Théry dans Couple, filiation et parenté aujourd'hui : le droit face aux mutations dela vie privée, Paris, Odile J acob - La Documentation française, 1998.

${ }^{67}$ Françoise Héritier, Masculin, Féminin. La pensée de la différence. Paris, Odile Jacob, 1996.

68 Voir Daniel Borrillo et Éric Fassin, Au-delà du PaCS. L'expertise familiale à l'épreuve de l'homosexualité, Paris, PUF, coll. « Politique d'aujourd'hui », deuxième édition, 2000.

69 Pour une analyse de ces formes de paternalisme, voir Michel Tort, La fin du dogme paternel, Paris, Flammarion, 2007.

70 Olivier Cayla et Yan Thomas, op. cit., p. 13.
} 
Daniel Borrillo, «La République des experts dans la construction des lois : le cas de la bioéthique », Histoire@Politique. Politique, culture, société, nº 14, mai-août 2011, www.histoire-politique.fr

Un autre argument invoqué par le courant néo-conservateur consiste à considérer que la libre disposition de soi mène nécessairement à la marchandisation du corps et à l'esclavage ${ }^{71}$. La gestation pour autrui est présentée comme une forme d'aliénation et une réification du corps de la femme, qui ne pourrait consentir à une telle pratique. Ainsi, lors des auditions devant l'Assemblée nationale, Sylviane Agacinski affirmait que « la gestation autorisée sera forcément rémunérée, faisant du ventre des femmes un instrument de production et de l'enfant lui-même une marchandise ${ }^{72} »$. Interrogée par la presse sur cette question, l'essayiste répondait : «J 'ai eu un enfant. Je sais ce que c'est qu'une grossesse. Cela représente une telle intimité organique que si l'on autorisait les mères porteuses, cela reviendrait à faire des organes d'une femme un four à pain. Ou d'une femme un animal destiné à la reproduction comme dans un élevage $^{73}$ » et lorsque cette professeure de philosophie est invitée réfléchir à l'homoparentalité, elle conclut: " On ne peut pas donner une filiation à un enfant à partir de deux personnes du même sexe, on est en plein délire. » La revendication des familles homosexuelles est ainsi renvoyée à la déraison et à la folie. La critique de la « frivolité mercantiliste » dérive très souvent vers une conception paternaliste et un essentialisme moral. Ainsi, le sociologue Éric Fassin a décelé dans les opinions de Sylviane Agacinski, un « féminisme de l'exception biologique ». Il souligne: " C'est abstraire la différence des sexes de l'histoire et de la politique. D'où le refus de toucher à la filiation. La nature biologique apparaît comme le dernier refuge contre la critique des normes. Ce naturalisme est une version laïcisée de la théologie du Vatican ${ }^{74}$.

Comme l'Église, la pensée néo-conservatrice s'oppose au mariage entre personnes de même sexe, à la filiation homoparentale, et à la gestation pour autrui, en prônant, pour ce faire, la primauté du biologique dans les liens familiaux et tant pis si pour cela il faut défaire le système civiliste du droit de la filiation et passer outre la volonté des individus concernés.

Comme le souligne Olivier Cayla, « il convient d'apercevoir dans toute son ampleur la signification politique précise que revêtent les concepts de "dignité humaine" et de "fonction anthropologique du droit", et de comprendre que leur orientation foncièrement hostile à toute idée de droit subjectif de l'individu les fait apparaître comme des instruments normatifs destinés à renier dans son principe le coeur des droits de l'homme du point de vue de la pensée politique moderne, c'est-à-dire à contester radicalement la liberté de l'individu dans la relation qu'il entretien avec luimême ${ }^{75} \gg$.

Le versant séculier du courant essentialiste trouve son origine dans la pensée aristotélicienne selon laquelle la Polis est antérieure à l'individu. C'est pourquoi, la tradition et tout ce qui comble le fossé entre l'individu et le monde apparaît comme

\footnotetext{
${ }^{71}$ Sylviane Agacinski-J ospin, Corps en miettes, Paris, Flammarion, 2009.

72 « Rapport sur l'évaluation de l'application de la loi du 6 août 2004 », op. cit., p. 146. Voir également le manifeste publié dans Le Figaro, signé par plusieurs personnalités (plusieurs d'entre elles avaient également manifesté contre le PaCS dans le passé) : « Mères porteuses : plaidoyer pour la défense des plus vulnérables », 12 mai 2009.

73 « Tout sur la mère », portrait de Sylviane Agacinski, Libération, 11 juin 2009.

74 Idem.

75 Olivier Cayla et Yan Thomas, op. cit., p. 13.
} 
Daniel Borrillo, «La République des experts dans la construction des lois : le cas de la bioéthique », Histoire@Politique. Politique, culture, société, nº 14, mai-août 2011, www.histoire-politique.fr

un argument privilégié des essentialistes. Au formalisme libéral du courant immanent, les tenants de l'éthique des convictions opposent une conception substantielle de la morale, véhiculée par une tradition donnée.

\section{Le « juste milieu » comme stratégie de l'expertise « neutre »}

Alors que la philosophie utilitariste, faisant confiance au contrat, donne une prééminence à la liberté individuelle, le courant métaphysique met l'accent sur les lois immuables (dogmes) extérieures à l'humain, auxquelles il doit se soumettre sous peine de mettre en danger l'ordre civilisationnel. La bioéthique à la française aime se présenter comme la voie moyenne entre ces deux pôles extrêmes. La sagesse serait donc à chercher entre le libéralisme anglo-saxon d'un côté, et la rigidité vaticane de l'autre. L'analyse d'Éric Fassin a bien mis en lumière la stratégie politique consistant à « dessiner une ambition pour notre temps : accompagner le progrès des mœurs sans bouleverser l'ordre social, rationaliser la société sans la dénaturer, bref, inventer une modernisation à visage humain. C'est tout le programme d'un orléanisme de gauche $^{76}$ ». Ainsi, ce n'est pas au nom de la sacralité de la vie mais au nom de la dignité humaine que certaines pratiques méritent d'être censurées. De même, le but thérapeutique, et non la liberté individuelle, permet de justifier l'accès à l'assistance médicale à la procréation réservé aux couples hétérosexuels. Or, la notion de stérilité du couple n'a aucune base scientifique. En effet, seuls les individus sont stériles et pas les couples. Cette fiction du couple infertile permet d'exclure les couples de même sexe et de justifier le remboursement des frais médicaux uniquement aux familles hétérosexuelles. Ce choix politique, présenté sous la forme d'un acte médical, perpétue une discrimination, cachée derrière une supposée raison thérapeutique. L'impératif « humaniste » et la « compassion clinique » viennent ainsi délimiter les contours du permis et de l'interdit.

Ainsi, la rhétorique «raisonnable» du «juste milieu» permet à la fois de se revendiquer d'une conception «moderne» de la famille tout en préservant sa structure traditionnelle. La stratégie est simple, il suffit, d'abord, de placer sur le même plan les traditions religieuses (même les plus réactionnaires) et les revendications progressistes de la société civile, pour se positionner ensuite comme le détenteur de la posture moyenne (raisonnable). Une experte, mobilisée aussi bien par la gauche socialiste que par la droite conservatrice, a su très bien incarner cette stratégie en opposant " d'un côté une interprétation des changements comme crise de la famille, indice et facteur d'une décadence morale et sociale et de l'autre une interprétation symétrique de la même évolution comme progrès des valeurs de liberté individuelle, d'authenticité et d'égalité ». Et face « au problème de la revendication croissante des homosexuels ${ }^{77}$ », le « juste milieu » a consisté à reconnaître pour ces couples uniquement le statut de concubins, afin de mieux leur refuser l'accès au

\footnotetext{
76 Éric Fassin, « P.A.C.S. socialista : la gauche et le "juste milieu" », Le Banquet, n 12, 1998.

${ }^{77}$ Irène Théry, Auditions de la Commission des Lois du Sénat, Les Rapports du Sénat n 481 1997-1998, p. 7 et suiv.
} 
Daniel Borrillo, «La République des experts dans la construction des lois : le cas de la bioéthique», Histoire@Politique. Politique, culture, société, nº 14, mai-août 2011, www.histoire-politique.fr

mariage et à la filiation ${ }^{78}$. Les détenteurs du «juste milieu » agissent en réalité à partir d'une éthique de conviction, d'un a priori qui consiste à croire que la différence des sexes est la base du régime des filiations. C'est pourquoi, face au caractère inéluctable des mouvements sociaux, l'inscription de l'origine biologique dans la loi est apparue, pour eux, comme la solution au «problème de l'homoparentalité ». En effet, avant cette revendication défendue par les gays et les lesbiennes, personne ne songeait à remettre en question le système civil de filiation qui était basé sur la volonté et les présomptions. Depuis, face au « danger » d'une désinstitutionalisation de la «différence des sexes», plusieurs experts (psychanalystes, sociologues, anthropologues, juristes...) n'ont cessé de dénoncer l'éloignement progressif du dispositif de filiation de sa base naturelle. Le retour au biologique permet de constituer un soubassement «vrai » sur lequel il serait désormais possible de construire des liens de filiation « fictifs », c'est-à-dire non fondés sur la reproduction charnelle. Ainsi, il s'est opéré à la fois (au sein de la pensée conservatrice) une remise en question de la loi de 1966 (qui autorise l'adoption monoparentale) et une critique de l'accouchement sous X. Cette double critique est accompagnée d'une demande de lever l'interdiction de l'anonymat en cas de don de gamètes ${ }^{79}$ et d'une inscription de l'origine biologique dans les actes de naissance en cas d'adoption plénière. C'est donc à condition de préserver quelque part la prééminence procréative de l'hétérosexualité que l'homosexualité pourra par la suite demander son inscription dans l'ordre des filiations ${ }^{80}$. Présentée comme une solution de bon sens, la question du biologique reprend une vigueur renouvelée ${ }^{81}$, d'une part en assurant la différence des sexes dans la filiation (tout enfant est issue des gamètes mâles et femelles) et d'autre part en fondant la norme non pas sur la volonté mais sur une figure de la transcendance (ordre symbolique de la différence des sexes). Se présentant comme détenteurs d'une pensée purement analytique (neutre), les tenants du « juste milieu » prétendent ainsi cacher leur combat d'arrière-garde ${ }^{82}$. En effet, au nom de la clinique psychanalytique ou de la nécessaire «différence des sexes », les experts « centristes » ne cessent de dénoncer (surtout depuis que la revendication homoparentale est apparue sur la scène publique) les mécanismes du droit civil qui ont permis la dissociation entre

\footnotetext{
78 Irène Théry, op. cit.

79 Geneviève Delaisai de Parseval a souligné que « le principe d'anonymat, en proclamant l'indifférence et l'interchangeabilité des gamètes, prive l'enfant, non seulement d'une partie de son histoire, mais aussi d'une partie d'humanité », « Rapport sur l'évaluation de l'application de la loi du 6 août 2004 », op. cit. p. 133

80 Irène Théry milite pour le droit de l'enfant non seulement à connaître ses origines biologiques mais à que ceux-ci soient inscrits dans la loi, sous peine de priver l'enfant de son histoire et de son hérédité. Une fois l'origine biologique instituée, l'homoparentalité pourrait trouver sa place dans l'ordre juridique. ${ }^{81}$ Le psychanalyste Christian Flavigny lors des auditions de la Mission d'information sur la révision des lois bioéthiques (18 février 2009) a critiqué l'adoption puisqu'elle se présente comme une forme d'enfantement effaçant ainsi le soubassement biologique de la filiation. Au nom de la psychanalyse, il se prononce contre l'homoparentalité et l'égalité des couples de même sexe.

82 Geneviève Delaisai de Parseval se « sent évidement plus proche » de la conception biologique de la filiation que de la conception moderne fondée plutôt sur la volonté : Famille à tout prix, Paris, Seuil, 2008, p. 198. Paradoxalement, bien qu'elle se présente comme « analyste», elle consacre le dernier chapitre (le plus volumineux) à faire des « Propositions au législateur »... autrement dit, sa finalité n'est pas descriptive et analytique mais bien prescriptive et normativiste.
} 
Daniel Borrillo, «La République des experts dans la construction des lois : le cas de la bioéthique», Histoire@Politique. Politique, culture, société, nº 14, mai-août 2011, www.histoire-politique.fr

sexe, reproduction et filiation : l'adoption plénière monoparentale, l'anonymat des donneurs et des donneuses de gamètes, l'accouchement sous $\mathrm{X}$, l'effacement des origines biologiques dans l'adoption, etc.

Face à l'inéluctabilité de l'homoparentalité, il est devenu urgent, aussi bien pour les conservateurs assumés que pour les détenteurs du «juste milieu », de procéder à un changement du dispositif juridique de la filiation pour permettre l'inscription de l'origine biologique de l'enfant dans la loi ${ }^{83}$. Aussi bien Irène Théry que Christine Boutin ne cessent de dénoncer la « violence » de l'anonymat des donneurs qui a fini par instituer «l'orphelinat légal » et a effacé le droit de l'enfant à connaître la «totalité de son histoire ${ }^{84}$ ». La députée catholique s'oppose à l'égalité pour les familles homoparentales au nom de l'ordre naturel tandis que la sociologue le faisait en invoquant l'ordre symbolique. Si les conséquences de leurs pensées respectives ne sont pas complètement identiques, la matrice idéologique est la même : résister au mouvement social afin de préserver la structure immuable de la filiation fondée sur la différence des sexes ${ }^{85}$. François Dagognet a raison d'affirmer que cette idéologie centriste a construit une nouvelle «prison physiologique » qui enferme la famille dans la vérité biologique ${ }^{86}$ et, plus généralement, elle a installé le débat bioéthique dans le «consensus et les fadeurs molles, plus insupportables encore que les condamnations prononcées par les intégristes, dominés par la culture ancienne ${ }^{87}$ ».

\section{Critique de la raison bioéthique}

La bioéthique apparaît en France comme une discipline de nature étatique, contrôlée par des experts désignés, à leur tour, par l'administration. Depuis l'origine, le dispositif est agencé de telle manière qu'aucun argument dans ce domaine n'échappe au contrôle de la puissance publique. En effet, l'administration, avec ses experts, contrôle l'ensemble du processus délibératif et normatif, à travers les comités de sages désignés par le président de la République, les rapports provenant des plus hautes instances de la magistrature (notamment le Conseil d'État), les J ournées nationales d'éthique, les agences publiques de biomédecine, les missions parlementaires et les forums de citoyens sous contrôle des experts agréés. De par la composition des institutions habilitées à se prononcer et la tutelle que l'État exerce sur ses experts, la production bioéthique est loin de jouir d'une véritable

\footnotetext{
83 Voir l'ouvrage collectif dirigé par Hugues Fulchiron, Mariage-conjugalité, parenté-parentalité, Paris, Dalloz, 2009.

${ }^{84}$ Audition d’Irène Théry du 10 juin 2008.

85 Irène Théry dans son rapport au gouvernement Jospin (1998) et dans ses écrits s'est toujours manifestée contre le mariage homosexuel, l'adoption plénière par des couples de même sexe, les techniques de reproduction assistée pour les familles monoparentales, l'anonymat des donneurs de gamètes, l'accouchement sous $\mathrm{X}$, la gestation pour autrui non thérapeutique... Irène Théry souhaite un changement du droit de la filiation afin qu'il ne cache plus « l'asymétrie et la complémentarité des sexes dans la procréation ». Il s'agit bien d'instituer la primauté de l'hétérosexualité dans l'ordre juridique de la famille, dans « Changer le regard sur la filiation », Le Monde, 24 janvier 2009.

86 François Dagognet, «La famille sans la nature: une politique de la morale contre le moralisme», dans Daniel Borrillo et Éric Fassin, Au-delà du PaCS, op. cit., p. 79 et suiv.

${ }^{87}$ François Dagognet, « Une bioéthique libertaire », Le Nouvel Observateur, 13 novembre 2003.
} 
Daniel Borrillo, «La République des experts dans la construction des lois : le cas de la bioéthique », Histoire@Politique. Politique, culture, société, nº 14, mai-août 2011, www.histoire-politique.fr

indépendance. En effet, la sélection des experts conduit au choix de personnalités qui bénéficient déjà d'une reconnaissance officielle, peu enclines à une attitude critique. L'avis des citoyens est circonscrit aux cercles désignés par l'État qui cherche plutôt à cautionner sa démarche en donnant une image de démocratie qu'à faire effectivement participer le peuple. La société civile n'est pas représentée dans les instances compétentes, et lorsque les autorités lui octroient un droit de parole, c'est toujours sous le contrôle minutieux de l’administration. Les États généraux de la bioéthique 88 sont, en ce sens, particulièrement significatifs de cette forme de délibération limitée : les citoyens sont là pour être éclairés par les savants (désignés par l'État comme tels) et lorsqu'ils sont invités à se prononcer sur le futur contenu des lois leurs points de vue coïncident parfaitement avec la pensée officielle ${ }^{89}$. De surcroît, comme le note Dominique Memmi, « depuis trente ans l'État s'est mis à intervenir massivement dans le rapport entre l'individu et sa destinée biologique ${ }^{90} »$. Depuis la création de l'Agence de biomédecine en 2005, la situation est devenue encore plus grave car elle a vocation à gouverner non seulement la pratique des greffes mais aussi la procréation et la génétique humaine. Outre son caractère éminemment étatique, la « mécanique » française repose sur une suspicion vis-à-vis de la délibération démocratique classique.

La méfiance à l'égard de la délibération démocratique renforce la figure de l'expert comme entité légitime dans l'élaboration des principes fondant la loi. Ainsi, la création du CCNE était basée sur l'idée que la complexité des questions bioéthiques nécessitait l'institutionnalisation d'une expertise permanente et régulière. Désormais, l'espace de délibération bioéthique par excellence s'est progressivement déplacé du Parlement vers certaines instances officielles spécialisées. La création du CNCE a produit la constitution d'une hiérarchie des arguments en octroyant aux membres de celui-ci le statut de "sages » capables d'émettre des avis plus éclairés que ceux provenant de la représentation populaire. Certes, les résolutions du CNCE ne sont pas contraignantes, il est toutefois difficile de s'y opposer lorsqu'elles apparaissent sur la scène politique comme le résultat, non seulement de la parole d'experts, mais aussi d'un consensus représentatif des sensibilités nationales. Or, la désignation des membres de ce comité n'a rien de démocratique. En effet, elle est, on l'a dit, effectuée par le président de la République sur proposition de l'administration et des forces religieuses. De surcroît, la société civile n'est pas représentée dans cette instance. Conscient de ce déficit, le gouvernement à mis en place les États généraux de la bioéthique $^{91}$, une sorte de forum citoyen chargé de définir les thèmes et de

88 Contrôlé par l'Agence de la biomédecine et le ministère de la Santé, ce type de délibération citoyenne s'est déroulée dans des forums régionaux, un forum national tenu à Paris le 23 juin 2009 et la mise en place d'un site Internet conçu sur la base de fiches d'information qui permettaient de mieux contrôler la parole publique.

89 Dans le rapport final, il est ainsi indiqué que l'assistance médicale à la procréation ne doit pas s'étendre à d'autres finalités que thérapeutiques excluant donc les couples de même sexe de cette forme de procréation.

90 Dominique Memmi, « Vers une confession laïque? La nouvelle administration étatique des corps », Revue française de science politique, 2000, vol. 50, n 1, p. 3-19.

${ }_{91}$ Décret $\mathrm{n}^{\circ}$ 2008-1236, du 28 novembre 2008, J O, 29 novembre 2008, p. 18233. 
Daniel Borrillo, «La République des experts dans la construction des lois : le cas de la bioéthique», Histoire@Politique. Politique, culture, société, nº 14, mai-août 2011, www.histoire-politique.fr

coordonner l'organisation du débat. La méthode choisie témoigne de l'absence de liberté délibérative: la discussion s'est engagée exclusivement entre les experts ( "grands témoins ») choisis par le comité de pilotage ${ }^{92}$ et le panel de quinze personnes présélectionnées par ce même comité dans trois villes (Marseille, Rennes et Strasbourg). Aucun débat avec la salle n'a eu lieu, seules les personnes du panel pouvaient questionner les spécialistes. De plus, sur le site internet créé pour l'occasion, certaines questions étaient déjà décidées à l'avance ${ }^{93}$. La Mission d'information sur la révision des lois bioéthiques de l'Assemblée nationale n'a pas fait preuve d'une plus grande ouverture. Elle n'a auditionné que des juristes conservateurs hostiles à toute évolution législative en la matière et aucun philosophe progressiste n'a été entendu par les représentants du peuple. L'appel constant à des valeurs comme la dignité humaine ou la non-instrumentalisation du fœetus ainsi que les dénonciations contre la « fascination technologique », « la marchandisation de la vie» ou le «tourisme procréatif » est le signe du manque de confiance en la délibération démocratique. La soumission à des principes considérés comme immuables semble donner l'impression d'un fondement plus solide à l'heure de justifier des choix sociétaux mais en réalité, elle ne fait que restreindre l'espace démocratique.

L'inexistence en France d'une force ecclésiastique capable d'influer sur l'opinion publique rend les positions anti-scientifiques encore plus efficaces car il est difficile de mettre en parallèle un discours ouvertement métaphysique et d'autres arguments qui, tout en partant des mêmes prémisses et en arrivant à des conclusions similaires, se présentent sous une forme séculaire et rationnelle. Certains juristes partent $d u$ postulat, sans jamais le démontrer, que le droit serait « asservi par le scientisme ${ }^{94}$ » et que la science est venue de manière intrusive occuper la place de la religion ${ }^{95}$. Le droit apparaît ainsi investi de la mission anthropologique d'instituer l'Humanité contre l'irruption invasive des sciences et des techniques ${ }^{96}$. La critique de la science et du marché permet de renouer avec une conception particulièrement prescriptive du droit, une démarche militante qui, en matière de bioéthique apparaît comme une réhabilitation du droit naturel. La pensée religieuse et le tenant de la pensée postmoderne se sont mobilisés pour la mise en place d'un dispositif légal particulièrement contraignant. Ainsi, un influent membre du comité d'éthique n’a pas cessé de dénoncer «les pouvoirs exorbitants de la recherche» qui «justifie l'assujettissement des humain, objets de science » et de prôner ardemment l'adoption

\footnotetext{
92 Présidé par le député catholique J ean Léonneti.

${ }^{93} \mathrm{La}$ formulation même des questions dans le site internet des États généraux ouvert au public était biaisée. Ainsi, il fallait répondre à l'interrogation suivante : « La médecine doit-elle répondre à tout prix au désir de l'enfant ? » ou « J usqu'à quel point chercher à avoir des enfants en bonne santé ? »...

94 J ean-René Binet, Droit et progrès scientifique. Science du droit, valeurs et biomédecine, Paris, PUF, 2002, p. 137.

${ }_{95}$ Muriel Fabre-Magnan et Philippe Moullier, La Génétique, science humaine, Paris, Belin, 2004.

96 Pour une critique de cette pensée conservatrice, voir Stéphanie Hennette-Vauchez, « Les rapports entre droit et science au prisme du droit de la bioéthique ou les larmes du crocodile », Cosmopolitiques, $n^{\circ} 8$, décembre 2004.
} 
Daniel Borrillo, «La République des experts dans la construction des lois : le cas de la bioéthique », Histoire@Politique. Politique, culture, société, nº 14, mai-août 2011, www.histoire-politique.fr

des lois afin d'éviter avec «l'emprise des corps », «le marché de la vie» ou «la brevetabilité du vivant ${ }^{97} »$, propres aux techno-sciences ${ }^{98}$.

Afin de justifier l'exclusion de certains individus (femmes célibataires, homosexuels...), le rapporteur de la mission parlementaire sur la révision des lois, J ean Léonetti, souligne : « L'accès médical à la procréation doit être examiné sous un angle médical, pas un angle sociétal. La médecine doit répondre à une pathologie, pas à une insatisfaction ou un désir. » Ce même argument apparaît dans les conclusions des États généraux de la bioéthique : «Que le désir d'enfant soit de fait, et de toute évidence, la raison fondamentale de recourir à l'assistance médicale à la procréation, n'implique pas de désigner la satisfaction de ce désir comme la finalité justifiant la mise en œuvre de cette pratique. S'il est admis que l'AMP a pour objectif de "remédier à l'infertilité dont le caractère pathologique a été médicalement diagnostiqué ou d'éviter la transmission à l'enfant ou à un membre du couple d'une maladie d'une particulière gravité" (alinéa 1 de l'article L 2141-2 du Code de santé publique), alors que ce n'est pas la satisfaction d'un désir d'enfant qui justifie l'usage de ces techniques. La satisfaction de ce désir est ici une conséquence possible de l'AMP, et non pas sa finalité. » Ce parallélisme n'est pas surprenant car le président du comité de pilotage des États généraux n'est autre que J ean Léonetti.

L'expertise en sciences humaines a produit une naturalisation de la famille hétérosexuelle. En effet, les lois bioéthiques se présentent en matière de procréation assistée comme un mécanisme tendant à palier la stérilité du couple ou à éviter la transmission d'une maladie génétique, c'est-à-dire comme un moyen de remédier à un dysfonctionnement de la nature. Pour ce faire, par le biais d'une fiction, les lois tentent d'imiter la nature. Ainsi, seuls peuvent avoir accès à l'AMP les couples de sexe différent en âge de procréer. Cette dimension « clinique » de la loi occulte un autre objectif : la reproduction de la conception naturalisée de la famille, fondée sur le couple hétérosexuel. En effet, la notion de « stérilité du couple » constitue, on l'a vu, une fiction. Une extension plus universaliste de cette fiction aurait permis d'accueillir en son sein également d'autres couples phénoménologiquement stériles comme les couples de même sexe. Ce ne fut nullement le cas et la fiction du couple stérile a permis de justifier « le choix de donner à l'enfant né d'une procréation non naturelle une famille normale et bien accordée ${ }^{99} »$. Les familles monoparentales et homoparentales ne trouvent pas leur place dans le dispositif bioéthique. Le psychanalyste Christian Flavigny lors de son audition parlementaire justifie cette exclusion comme suit : «L'enfant né de l'union d'un homme et d'une femme, sur le

\footnotetext{
97 Catherine Labrusse-Riou, « La maîtrise du vivant : matière à procès », Pouvoirs, n 56, 1991, PUF, p. 87 et suiv.

98 Dans un article du journal Le Monde (25 mars 1992), cosigné par Bernard Edelman, Marie-Angèle Hermitte, J acques Testart et Monique Vacquin, les auteurs appellent à une interdiction du diagnostic préimplantatoire.

99 Conseil d'État, Les lois bioéthique : cinq ans après, 1999, p. 19.
} 
Daniel Borrillo, «La République des experts dans la construction des lois : le cas de la bioéthique », Histoire@Politique. Politique, culture, société, nº 14, mai-août 2011, www.histoire-politique.fr

plan biologique mais aussi psychique. C'est tout l'enjeu de la différence des sexes (...). L'enfant aspire à se sentir advenu d'une union crédible qu'il symbolise ${ }^{100}$. »

Les techniques de procréation artificielle, au lieu de permettre l'accomplissement d'une égalité des sexes (hommes-femmes) et des sexualités (homo-hétéro), ont servi à promouvoir une conception traditionaliste de la famille. La femme est celle qui accouche (acte naturel), le père est le mari de la mère (acte culturel) et la filiation est basée sur l'acte sexuel (biologique). Si la science avait permis de « débiologiser » et « dénaturaliser » la famille, grâce aux différentes techniques de dissociation de la sexualité et la reproduction, le débat bioéthique a permis de dénoncer cette dissociation au nom de la « vérité » biologique et du droit à connaître les origines. Ce retour au biologique apparaît toutefois comme purement instrumental, il est là uniquement pour garantir la prééminence de la famille classique définie par le Conseil d'État par la formule « deux parents, pas un de plus, pas un de moins ». Et si bientôt pourront se fondre deux cellules reproductrices mâles ou femelles (deux spermatozoïdes ou deux ovocytes) pour déclencher le cycle embryonnaire, cette possibilité est d'ores et déjà dénoncée par les défenseurs de la vérité biologique et du droit à connaître les origines se repliant sur une autre objection : l'enfant sera privé d'un père ou d'une mère. Derrière les arguments invoqués se cache bien la défense du modèle canonique de la famille car, comme l'a brillamment démontré Rémi Lenoir, la famille nucléaire (maman-papa-enfant) est devenue l'élément naturel à partir duquel il est possible de penser « naturellement » l'individu, la société et le monde ${ }^{101}$.

\section{Conclusion : le biopouvoir des experts}

Pour les sociétés démocratiques, l'impartialité morale de l'État constitue la garantie de la liberté des individus. Ce principe se fonde sur l'idée selon laquelle l'État (et le droit) est neutre à l'égard des conceptions substantielles du bien et se limite à énoncer le juste et à garantir, notamment par l'intervention prétorienne, l'équilibre des relations humaines afin d'éviter les abus ${ }^{102}$. Le rôle de la puissance publique n'est ni de rendre les citoyens vertueux ni de promouvoir des fins particulières, mais seulement de garantir les libertés fondamentales. Contrairement à l'État paternaliste, l'État démocratique ne se substitue pas aux choix des individus. C'est à eux de déterminer leur manière de vivre et de décider de ce qui est bon pour la réalisation de leurs objectifs vitaux. L'État est ainsi appelé à éviter de prendre position dans le conflit des conceptions du monde lesquelles doivent rester confinées à la sphère privée. Ce qui fait qu'une société soit juste, « ce n'est pas le telos, le but ou la fin qu'elle poursuit, mais précisément son refus de choisir à l'avance parmi des buts et des fins concurrents 103 ». Si l'État ne doit pas s'immiscer à certains moments (liberté négative), il doit s'engager néanmoins à intervenir à d'autres moments (liberté

\footnotetext{
100 Rapport de la Mission d'information sur la révision des lois bioéthiques, p. 52.

101 Rémi Lenoir, Généalogie de la morale familiale, Paris, Seuil, 2003.

102 J ohn Rawls, Libéralisme politique (1993), (trad. C. Audard), Paris, PUF, 1995.

103 Michael Sandel, «La république procédurale et le moi désengagé », dans André Berten, Pablo da Silveira et Hervé Pourtois (textes réunis et présentés par), Libéraux et communautariens, Paris, PUF, 1997, p. 255.
} 
Daniel Borrillo, «La République des experts dans la construction des lois : le cas de la bioéthique », Histoire@Politique. Politique, culture, société, nº 14, mai-août 2011, www.histoire-politique.fr

positive). Il ne doit pas décider à la place de l'individu mais garantit l'exercice des droits individuels de manière égalitaire. Tout acte librement accompli dès lors qu'il est dépourvu de conséquences négatives pour autrui doit rester en dehors de la sanction juridique. Cependant, l'État ne peut pas se contenter d'énoncer ce principe, il doit également s'assurer que la liberté est matériellement effective aussi bien dans les relations que l'individu entretient avec lui-même (autonomie de la volonté) qu'avec les autres (équilibre contractuel). Ainsi, lorsqu'il résulte d'un choix libre et informé tout acte qui nelèse pas autrui doit être considéré comme moral. Une femme doit être non seulement libre d'interrompre une grossesse mais aussi de disposer de son corps pour une gestation pour autrui. Puisque l'idée de « couple stérile » est une fiction qui permet l'accès médical à la procréation, elle peut s'élargir aussi bien aux femmes célibataires qu'aux couples de même sexe afin de permettre à l'ensemble des individus (célibataires, en couple, hétérosexuels ou homosexuels) de mener à bien leurs projets parentaux.

Comme les Églises, la pastorale étatique décide qui peut procréer et qui ne doit pas le faire et ceci non pas au nom des lois divines mais au nom de l'intérêt de l'enfant à naître, de la cohérence des structures familiales ou des repères anthropologiques. Peu importe que les femmes célibataires et les couples de même sexe voient disparaître leurs projets parentaux, ils n'ont qu'à faire le deuil de l'enfant, même s'ils disposent des compétences morales et matérielles requises pour devenir parents. Souvent, comme nous avons essayé de le démontrer tout au long de notre article, la bioéthique ne garantit pas l'épanouissement de l'ensemble de la population mais constitue une arme redoutable d'immixtion des pouvoirs publics dans la relation privée de l'individu à son destin physique ${ }^{104}$. Dans ces cas, il faudrait cesser de parler de bioéthique pour utiliser le terme de « biopouvoir » en tant que version moderne de la pastorale chrétienne, c'est-à-dire, comme le soulignait Michel Foucault, d'un pouvoir qui ne s'exerce plus sur les âmes mais sur le corps et la vie des individus; d'une politique qui fait de l'espèce humaine l'objet d'une stratégie générale de domination. L'espace ouvert par le débat bioéthique a été investi par des courants conservateurs, lesquels au nom de la « dignité humaine» ne font que réactualiser le vieux combat contre la modernité et plus particulièrement contre la liberté de disposer de soi qui est à l'origine du dispositif contemporain des droits de l'homme. Le corps humain devient ainsi un espace public à contrôler. Et, la bioéthique se transforme en biopouvoir lorsque l'État et ses experts se substituent aux choix vitaux des individus prétendant savoir mieux qu'eux-mêmes ce qui leur convient pour la réalisation de ces mêmes choix.

\section{L'auteur}

Daniel Borrillo est juriste, maître de conférences en droit privé à l'Université de Paris Ouest-Nanterre/La Défense, chercheur associé au CERSA (Centre d'études et

104 Didier Fassin et Dominique Memmi (dir.), Le Gouvernement des corps, Paris, Éditions de l'EHESS, 2004. 
Daniel Borrillo, «La République des experts dans la construction des lois : le cas de la bioéthique », Histoire@Politique. Politique, culture, société, nº 14, mai-août 2011, www.histoire-politique.fr

recherches en sciences politiques et administratives, CNRS). Auteur de plusieurs ouvrages parmi les plus récents : Bioéthique (Dalloz 2011), Le droit des sexualités (PUF 2010), Halde: actions, limites et enjeux (La Documentation française, 2007). Daniel Borrillo anime actuellement trois séminaires de doctorat sur le droit de l'égalité et la non-discrimination dans le CERSA, sur le droit du genre et des sexualités dans le Master des droits de l'homme de l'Université de Paris Ouest et sur le droit pénal de l'environnement dans cette même université. Il est professeur invité à l'Université Carlos III de Madrid et la Pontificia Universidade Catolica de Rio de Janeiro.

\title{
Résumé
}

La bioéthique est née en France comme un savoir d'État. La place des experts dans la production des lois dans cette matière est d'une particulière importance non seulement par la prolifération de débats publics mais aussi par la production des rapports officiels. Paradoxalement, ce ne sont pas les experts en sciences médicales qui sont les plus mobilisés et influents dans la production des lois mais les experts en sciences humaines conçues non pas comme un savoir descriptif mais comme une discipline prescriptive. Cette situation fait de la bioéthique un dispositif plus proche de la théologie que des sciences sociales ce qui explique que la bioéthique apparaît plus comme une règle morale s'imposant aux individus que comme un savoir permettant de mieux les éclairer dans leur choix vitaux.

\begin{abstract}
Bioethics was institutionalized in France as a State Science. The place of experts in the legal production is particularly important. The proliferation of debates and the prolific production of official reports place the specialist at the heart of the political decision system. Paradoxically, it is not the expert in biological sciences or the physician who is at the centre of the production system of laws but the social scientist, but social sciences do not appear as a descriptive knowledge but a prescriptive discipline, which makes the expert closer to the theologian than to sociologist and the bioethics closer to theology than to explanatory knowledge able to inform the patients and to assist them in their decisions.
\end{abstract}

Mots clés: bioéthique, expertise, dignité humaine, ordre symbolique, antilibéralisme.

Keys words : Bioethics, expertise, human dignity, symbolic order, anti-liberalism.

Pour citer cet article : Daniel Borrillo, «La République des experts dans la construction des lois : le cas de la bioéthique », Histoire@Politique. Politique, culture, société, n 14, mai-août 2011, www.histoire-politique.fr 